\title{
EDITORIAL
}

\section{Restructuring the UNIFESP Nursing Graduate Program}

The graduate program evaluation system implemented by CAPES has been contributing with the development of a continuous analysis culture regarding the processes and outcomes of human resource formation, knowledge production, and technology innovation in the country.

In this setting, of a quest for excellence, the nursing graduate programs have made efforts to identify strengths and weaknesses, with a view to develop strategies that would consolidate and enhance successful activities and promote opportunities of transformation to improve the processes that are in their consolidation phase.

Hence, the UNIFESP nursing graduate program has proactively performed self-evaluation seminars, reflecting about the scientific production paradigms in nursing, which allowed for an epistemological analysis of the program's proposals for education and knowledge production. For this analysis, it was also taken into consideration the information from the Coleta CAPES reports in the last six years (200 to 2006) and the recommendations for improving the proposals by the nursing area evaluation committees.

The result of this evaluation process generated changes on the program proposal, which was structured by the axes of health-disease process and life cycle. It was found that the area of concentration: Nursing, Care and Health reflects the program's production, covering studies about theories, concepts, principles, methods and instruments that guide the practice and use of technologies in nursing management, coordination and care for the development of the health area in both the individual and collective scopes.

Thus, the program's mission was conceived as that of becoming a center of excellence for the formation of researchers, leaders, and highly capacitated professionals to promote nursing and health science advancement, with emphasis on multidimensional approach to people and groups considering their different expressions. Through research, education, and practice teachers and students seek to promote people's health within the context of the current health needs of the population and of the future generations, in agreement with the Single Health System principles.

The program is founded on the thought that humans have prerogatives and rights that must be respected. The right to life and health is essential for one's self-fulfillment. From this perspective, assistance is focused on the health care needs in the individual and collective scope by means of knowledge that integrates the bases: scientific, interpretative/personal, critical, ethical, aesthetical, and sociopolitical. These foundations guide knowledge production that would contribute with an innovating and transforming professional practice.

The curricular structure and research lines developed in consonance with the fundamental concepts and program objectives and adopts interdisciplinarity as its principle, integrating the knowledge that strengthen Nursing and Health.

This collective work permitted faculty and graduates to reflect about this new program concept, thus allowing the elaborated transformations to culminate in a new understanding of how much each subject and field of investigation are interrelated and in consonance with the proposed objectives for the formation of masters and doctorates.

Dra. Janine Schirmer, Ph.D. Mavilde da Luz Gonçalves Pedreira, Ph.D. Coordinators of the UNIFESP Nursing Graduate Program

Mariana Fernandes de Souza, Ph.D. Maria Gaby Rivero de Gutiérrez, Ph.D.

Coordinators of the Restructuring Committee of the UNIFESP Nursing Graduate Program 